\title{
An aerodynamic approach in soil hydraulic conductivity estimation for investigating soil erosion degree
}

\author{
VASYUKOV SERGEY ${ }^{1} \&$ SIROTKIN VYACHESLAV ${ }^{2}$ \\ 1 Federal Service for State Registration, Cadastre and Cartography, PO Box 428023, Elmenya st. house 12, of. 52, \\ Cheboksary, Russia \\ svasyukov@rambler.ru \\ 2 Kazan Federal University, Geography and Cartography Department, Institute of Ecology and Geography, \\ 18 Kremlevskay St., Kazan, Russia, 420008 Kazan, Russia \\ sirotkin67@,rambler.ru
}

\begin{abstract}
We propose a new method for determining the degree of erosion for zonal soils of the East European Plain. This new approach uses soil porosity and filtration to determine a coefficient of aerodynamic similarity. We evaluated the degree of soil erosion on ranges of the major zonal soils of the eastern part of European Russia by applying this new method. Based on these data, we developed a diagnostic scale to determine the extent of soil erosion in this area.
\end{abstract}

Key words surface erosion; soils; filtration coefficient; porosity; diagnostic scales.

\section{INTRODUCTION}

Surface soil erosion is a ubiquitous feature of our landscape. Water flow from rain, snow and spring thaw transport and deposit soil, often unpredictably. Most surface soil erosion occurs in agricultural areas. As no universal criteria for the determination of the severity of erosion exists, we generated a method by which soil surface erosion may be quantified. A number of methods for soil porosity measurement are known: Richards (1931), Kraus et al. (1953), Sirotkin \& Ginzburg (1971), Dullien (1991), and Stewart et al. (2013). These methods may be performed in a laboratory environment, or in the field, by determining the capillary porosity or aeration (Onishchenko, 1986). In contrast to these methods, we propose a new method of measuring total porosity that does not require the solid phase density measurement. Therefore, our new method may be used for undisturbed soils without excessive water content.

\section{METHOD DEVELOPMENT}

This method is based on the isothermal expansion of air trapped in soil pores when the soil sample is set within a vacuum. A sample of undisturbed soil with a set volume, $V_{\text {soil }}$, is placed in the vessel 1 of $V_{1}$ volume. Vessel 1 is connected by a vacuum gauge to vessel 2 of a known volume, $V_{2}$. Vessel 2 is then connected to an atmosphere or a vacuum pump. Both vessels are then sealed. Vessel 1 is split-off by taps set in vessel 2 at $p_{2}$ vacuum. Vessel 1 and the sample have $p_{1}$ pressure, equal to 1 atmosphere. Both vessels are then connected and the expansion of air from the sample contained within vessel 1 flows into vessel 2. A common pressure $p$ will then be established for the system.

Using the Clapeyron-Mendeleev law, the following formulae are obtained:

$$
\begin{aligned}
& p_{1} V_{1}=\frac{m_{1}}{\mu} R T_{1}-\text { for the first vessel } \\
& p_{2} V_{2}=\frac{m_{2}}{\mu} R T_{2}-\text { for the } 2 \text { nd vessel } \\
& p\left(V_{1}+V_{2}\right)=\frac{m_{1}+m_{2}}{\mu} R T-\text { for the whole system }
\end{aligned}
$$

where $m_{1}$ is the mass of gas in the first vessel; $m_{2}$ is the mass of gas in the second vessel; $R$ is a universal gas constant; $T_{1}, T_{2}, T$ are the temperatures in the first and second vessels and in the entire system; $\mu$ is the molar mass of air.

Assuming the process is an isothermal one, i.e.: 


$$
T_{1}=T_{2}=T
$$

equations (1), (2), (3) express the pressure $p$, which is read on the vacuum meter after the vessel connection:

$$
p=\left(p_{1} V_{1}+p_{2} V_{2}\right) /\left(V_{1}+V_{2}\right)
$$

$V_{I}$ volume consists of the soil-ground volume, $V_{\text {por }}$, and volume $V_{1}$, which is determined experimentally on the same apparatus by replacing the non-porous soil-ground sample with a nonporous body of the same volume. After transformation, the following formula may be obtained:

$$
V_{\text {por }}=\frac{V_{2}\left(p_{2}-p\right)+V_{1}\left(p_{2}-p\right)}{p-p_{1}}
$$

whereby $V_{\text {por }}$ is the pore volume in the sample occupied by air.

At a parallel measurement of humidity, the volume of moisture within the soil pores, $V_{\text {water, }}$ may be calculated. Thus, the total porosity of the sample makes:

$$
P=\left(V_{\text {pores }}+V_{\text {water }}\right) / V_{\text {soil. }}
$$

Substituting the obtained value of porosity from equation (7) and the experimentally determined value of the bulk density, it is possible to estimate the density of the solid phase for an undisturbed sample:

$$
p_{s}=p_{v} /(1-P)
$$

In our study, soil samples of about $500 \mathrm{~cm}^{3}$ were collected. Air pressure transmitted through the sample was measured with a vacuum gauge from the exhaust meter mark of $0.05 \mathrm{~atm}$, which allowed measurement of the porosity of the same sample with five repeats giving an average relative error of less than $1 \%$, and for different specimens of the same depth at a distance of $0.5 \mathrm{~m}$ apart with a maximum error of about $2 \%$.

The sample humidity was determined by a thermostatic-gravimetric method. The proposed method of measuring the porosity and density of the soil solid phase is used in the field conditions with a portable vacuum pump.

The infiltration coefficient was determined using computational methods based on the measurements of other variables, the determination of which, according to the authors, is more reliable, convenient and secure with methodological and technical aspects.

We used aerodynamic method of hydraulic conductivity determination (coefficient of hydraulic conductivity) for a generalized description of the relationship between permeability factor and humidity for large groups of dispersed systems.

Continuous media stream as it moves through a porous system is determined by Darcy's law:

$$
Q=K \frac{\Delta P}{\Delta x} S_{S}
$$

Using an idealized model (Fig. 1) of soil partials, a soil sample may be replaced in calculation by a direct cylinder tube of an effective radius, the length of which is equal to the sample length, and the energy loss when the continuous medium is flown through it is equal to the energy losses at the flow of the same medium through the same sample. For this tube current the flow is determined by the Poiseuille equation:

$$
Q=\frac{\pi}{8 \eta} \frac{\Delta P}{\Delta x} R_{e f}^{4}
$$

Equation (10) corresponds to the case when the soil-ground portion of the soil-ground space is occupied by moisture, i.e. the numerical values of $Q, \Delta P, R_{e f}$ are obtained from the air flow experiment described above. For the case of moisture lack in pores, the same equation will be as follows:

$$
Q_{0}=\frac{\pi}{8 \eta} \frac{\Delta P}{\Delta x} R_{0 e f}^{4}
$$

where the values of $Q_{0}, R_{0 e f}$ correspond to the dry sample. When the sample humidity is changed the air consumption rate flowing through it decreases with moisture volume increase. This flow rate change is determined by the expression formulated for a special case with the measured value $R_{e f}$. 


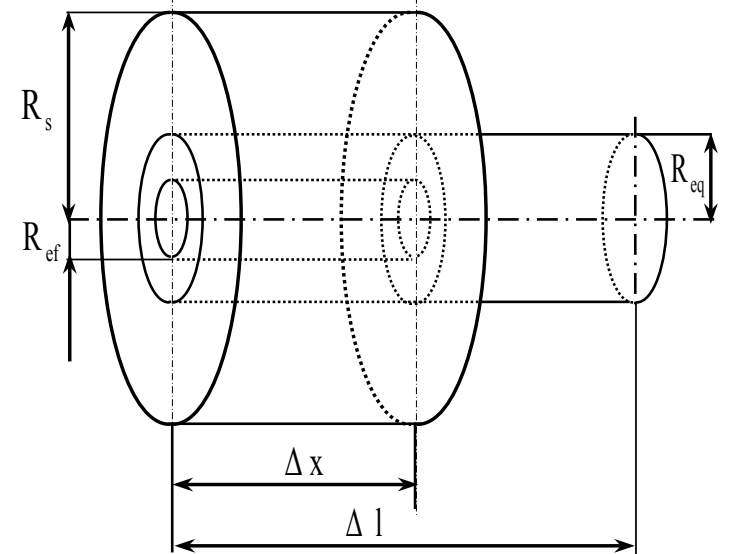

Fig. 1 An idealized model of soil. Rs is the radius of the sample, $R_{\mathrm{ef}}$ is the effective radius, the radius of the sample flow tube, where gas flow $Q$ is equal to the gas flow through the sample at the same pressure gradient, Req- equivalent radius, $\Delta \mathrm{x}$ is sample length, $\Delta 1$ is the length of the tube with equivalent radius, the energy losses are equal to the flow in the tube of the effective radius and thus losses in the air flow through the sample.

$$
\Delta Q=Q_{0}-Q=\frac{\pi}{8 \eta} \frac{\Delta P}{\Delta x}\left(R_{0 e f}^{4}-R_{e f}^{4}\right)
$$

The same change of consumption $\Delta Q$ can be formally represented in the form of Darcy's law:

$$
\Delta Q=K^{\prime} \frac{\Delta P}{\Delta x} S_{S}
$$

where $\Delta Q$ is an air consumption rate through the cylindrical layer of thickness $\Delta h=R_{0 e f}-R_{e f} ; K^{\prime}$ is the air permeability coefficient through this layer.

Equating (12) and (13) we obtain the following formula:

$$
\frac{\pi}{8 \eta}\left(R_{0 e f}^{4}-R_{e f}^{4}\right)=K^{\prime} S_{S}
$$

When the air permeability coefficient $K^{\prime}$ is expressed from (14), we obtain:

$$
K^{\prime}=\frac{\pi}{8 \eta} \frac{\left(R_{0 e f}^{4}-R_{e f}^{4}\right)}{S_{S}}
$$

The air consumption through the flow tube of a measured effective radius corresponding to the wet sample $R_{e f}$, and the air flow through the flow tube of the same length with an effective radius $R_{0 e f}$, corresponding to dry sample are different and described by the following equations:

$$
\begin{aligned}
& \frac{\Delta V}{\Delta t}=\frac{\pi}{8 \eta} \frac{\rho g \Delta h}{\Delta x} R_{e f}^{4} \\
& \frac{\Delta V}{\Delta t_{0}}=\frac{\pi}{8 \eta} \frac{\rho g \Delta h_{0}}{\Delta x} R_{0 e f}^{4}
\end{aligned}
$$

Expressing the corresponding effective radii from equations (16) and (17) the following equations are obtained:

$$
\begin{aligned}
& R_{e f}^{4}=\frac{\Delta V 8 \eta \Delta x}{\Delta t \Delta h \rho g \pi} \\
& R_{0 e f}^{4}=\frac{\Delta V 8 \eta \Delta x}{\Delta t_{0} \Delta h_{0} \rho g \pi}
\end{aligned}
$$

or, given that the air volume of $\Delta V$ is a constant, we obtain:

$$
\frac{R_{e f}^{4}}{R_{\text {Oef }}^{4}}=\frac{\Delta t_{0} \Delta h_{0}}{\Delta t \Delta h}
$$


which includes two uncertain values, $R_{0 e f}$ and $\Delta t_{0} \Delta h_{0}$, which are used to define the concept of equivalent radii.

Equivalent radius in this model can be defined as the radius of a single cylindrical pore with the length of $\Delta x$, the volume of which is equal to the total pore volume of the sample.

Writing the Poiseuille equations for flow tubes of equivalent radii $R_{0 e q}$ and $R_{e q}$, aerodynamically, similar to the relevant flow tubes with $R_{0 e f}$ and $R_{e f}$ radii of the same length $\Delta l$, we obtain the following formulations:

$$
\begin{aligned}
& Q=\frac{\pi}{8 \eta} \frac{\Delta P}{\Delta l} R_{e q}^{4} \\
& Q_{0}=\frac{\pi}{8 \eta} \frac{\Delta P}{\Delta l} R_{0 e q}^{4}
\end{aligned}
$$

Using arguments similar to the above, we obtain:

$$
\frac{R_{e q}^{4}}{R_{0 e q}^{4}}=\frac{\Delta t_{0} \Delta h_{0}}{\Delta t \Delta h}
$$

where:

$$
\Delta t_{0} \Delta h_{0}=\Delta t \Delta h \frac{R_{e f}^{4}}{R_{0 e f}^{4}}
$$

Equivalent radii are found from the following equations:

$$
\begin{gathered}
P=\frac{R_{e q}^{2}}{R_{S}^{2}} \\
P_{0}=\frac{R_{0 e q}^{2}}{R_{S}^{2}}
\end{gathered}
$$

where $R_{s}$ is the sample radius, $P$ and $P_{0}$ are the porosity in wet and dry conditions, respectively. $P$ is determined experimentally, while $P_{0}$ is calculated by:

$$
P_{0}=W_{v}+P \text {, }
$$

where $W_{v}$ is volumetric moisture in unit fractions, i.e. the value measured experimentally.

Substituting the values of $R_{e q}$ and $R_{0 e q}$ from equations (25) and (26) into (27), we obtain:

$$
\Delta t_{0} \Delta h_{0}=\Delta t \Delta h \frac{P^{2}}{P_{0}^{2}}
$$

$\Delta t, \Delta h$ and $P$ are measured during the experiment for specific humidity value $W_{v}$.

Using the relation analogous to the expression (28) the current $\Delta t_{i} \Delta h_{i}$ can be obtained from $P_{i}$ :

$$
\Delta t_{i} \Delta h_{i}=\Delta t_{0} \Delta h_{0} \frac{P_{0}^{2}}{P_{i}^{2}}=\Delta t \Delta h \frac{P^{2}}{P_{i}^{2}}
$$

Denoting the air permeability coefficient measured in speed units via $K_{l}^{\prime}$ and taking into account the fact that it is associated with $K^{\prime}$ the air permeability coefficient obtained from equation (15) by the relation:

$$
K_{l}^{\prime}=K^{\prime} \rho g
$$

from (15), (18), (19) and (29) we obtain the following:

$$
K_{l i}^{\prime}=\frac{\Delta V \Delta x}{\Delta t \Delta h S_{S} P^{2}}\left(P_{0}^{2}-P_{1}^{2}\right)
$$

The relationship between the air permeability coefficient and hydraulic conductivity coefficient may be obtained from same laws of Darcy and Poiseuille. For the case of pore complete filling with a continuous medium, these laws may be formulated in the following way: 


$$
\begin{array}{ll}
Q_{1}=K_{1} \frac{\Delta P}{\Delta x} S_{S}, Q_{1}=\frac{\pi}{8 \eta_{1}} \frac{\Delta P}{\Delta x} R_{e f}^{4} & \text { for air } \\
Q_{2}=K_{2} \frac{\Delta P}{\Delta x} S_{S}, Q_{2}=\frac{\pi}{8 \eta_{2}} \frac{\Delta P}{\Delta x} R_{e f}^{4} & \text { for water }
\end{array}
$$

where $Q_{1}$ and $Q_{2}$ are the consumption rate of air and water, respectively; $K_{1}$ and $K_{2}$ are the filter coefficients; $\eta_{1}$ and $\eta_{2}$ are the viscosity coefficients. For both media the relations may be formulated:

$$
\frac{\pi}{8 \eta_{1}} R_{e f}^{4}=K_{1} S_{S} \text { and } \frac{\pi}{8 \eta_{2}} R_{e f}^{4}=K_{2} S_{S}
$$

from which we obtain the relation between the filter coefficients highlighting and equating constants:

$$
\frac{K_{!}}{K_{2}}=\frac{\eta_{2}}{\eta_{1}}
$$

This ratio serves as a scale factor when replacing one continuous medium by another. Then, for the case of water flow through an unsaturated sample, the hydraulic conductivity coefficient is determined by an expression similar to (31):

$$
K_{2 i}^{\prime}=\frac{\Delta V \Delta x}{\Delta t \Delta h S_{S} P^{2}} \frac{\eta_{1}}{\eta_{2}}\left(P_{0}^{2}-P_{1}^{2}\right)
$$

Since $P_{i}=P_{0}-W_{v i}$, where $W_{v i}$ is the current value of volume moisture in unit fractions, then equation (36) may be formulated as:

$$
K_{2 i}^{\prime}=\frac{\Delta V \Delta x}{\Delta t \Delta h S_{S} P^{2}} \frac{\eta_{1}}{\eta_{2}}\left[P_{0}^{2}-\left(P_{0}-W_{v i}\right)^{2}\right]
$$

where $\Delta V, \Delta x, \Delta t, \Delta h, S_{s}, P$ are measured values; $\eta_{1}$ and $\eta_{2}$ are constants; $W_{v i}$ is a defined value.

Equation (37) expresses the relation between the coefficient of hydraulic conductivity and volumetric moisture. The boundary conditions of equation (33) are: $P_{i}=0$ is the case of complete filling of a continuous medium; $P_{i}=P_{0}$ when the pores are completely free. For the first case, the coefficient of the hydraulic conductivity coefficient is equal to the infiltration coefficient:

$$
K_{2}^{\prime}=\frac{\Delta V \Delta x}{\Delta t \Delta h S_{S} P^{2}} \frac{\eta_{1}}{\eta_{2}} P_{0}^{2}
$$

For the second (dry sample), $K_{2}^{\prime}=0$.

\section{APPLICATION AND RESULTS}

Based on the above facts, we have examined, under field conditions, the basic zonal soils of the Eastern European Plains with various erosion degrees and used the proposed methodology to determine their degree of erosion: soddy-podzolic soils, gray forest soils, leached black earth, based on the infiltration coefficient, porosity and bulk density changes (Tables $1,2,3)$. It is possible that in other areas such data may differ from those listed below (due to the change of soil-

Table 1 Diagnostic table to determine the erosion of soddy-podzolic soils.

\begin{tabular}{lllll}
\hline Soil erosion degree & $\begin{array}{l}\text { Filtration } \\
\text { coefficient } \\
\text { change (\%) }\end{array}$ & $\begin{array}{l}\text { Porosity } \\
\text { change (\%) }\end{array}$ & $\begin{array}{l}\text { Volume weight } \\
\text { change (\%) }\end{array}$ \\
\hline Absent & - & - & - & - \\
Weak & $0-(-40)$ & $0-(-15)$ & $0-(-2)$ & $0-(+10)$ \\
Average & $-40-(-80)$ & $-15-(-25)$ & $-2-(-25)$ & $+10-(+20)$ \\
Strong & $-80-(-95)$ & $-25-(-50)$ & $-25-(-50)$ & $+20-(+30)$ \\
\hline $\begin{array}{l}\text { Distance from the } \\
\text { surface }\end{array}$ & $0-20$ & $0-20$ & $20-40$ & $0-20$ \\
\hline
\end{tabular}


An aerodynamic approach in soil hydraulic conductivity estimation for investigating soil erosion degree 71

Table 2 Diagnostic table to determine the erosion of gray forest soils.

\begin{tabular}{|c|c|c|c|c|c|c|c|c|}
\hline \multirow{2}{*}{$\begin{array}{l}\text { Soil erosion } \\
\text { degree }\end{array}$} & \multicolumn{3}{|c|}{$\begin{array}{l}\text { Filtration coefficient } \\
\text { change }(\%)\end{array}$} & \multicolumn{3}{|c|}{$\begin{array}{l}\text { Porosity } \\
\text { change }(\%)\end{array}$} & \multicolumn{2}{|c|}{$\begin{array}{l}\text { Volume weight } \\
\text { change }(\%)\end{array}$} \\
\hline & - & - & - & - & - & - & - & - \\
\hline Weak & $0-(-10)$ & $0-(-25)$ & $0-(-40)$ & $0-(-2)$ & $0-(-1)$ & $0-(-1)$ & $0-(+2)$ & $0-(+2)$ \\
\hline Average & $-10-(-25)$ & $-25-(-35)$ & $-40-(-50)$ & $-2-(-4)$ & $-1-(-5)$ & $-1-(-3)$ & $+2-(+7)$ & $+2-(+4)$ \\
\hline Strong & $>(-25)$ & $>(-35)$ & $>(-50)$ & $>(-4)$ & $>(-5)$ & $>(-3)$ & $>(+7)$ & $>(+4)$ \\
\hline $\begin{array}{l}\text { Distance from } \\
\text { the surface }\end{array}$ & $0-20$ & $20-40$ & $40-60$ & $0-20$ & $20-40$ & $40-60$ & $0-20$ & $20-40$ \\
\hline
\end{tabular}

Table 3 Diagnostic table to determine the erosion of leached chernozem.

\begin{tabular}{|c|c|c|c|c|c|c|}
\hline $\begin{array}{l}\text { Soil erosion } \\
\text { degree }\end{array}$ & \multicolumn{2}{|c|}{$\begin{array}{l}\text { Filtration coefficient } \\
\text { change }(\%)\end{array}$} & \multicolumn{2}{|l|}{$\begin{array}{l}\text { Porosity } \\
\text { change (\%) }\end{array}$} & \multicolumn{2}{|c|}{$\begin{array}{l}\text { Volume weight } \\
\text { change }(\%)\end{array}$} \\
\hline Absent & - & - & - & - & - & - \\
\hline Weak & $0-(-50)$ & $0-(-50)$ & $0-(-18)$ & $0-(-18)$ & $0-(+13)$ & $0-(+8)$ \\
\hline Average & $-50-(-95)$ & $-50-(-95)$ & $-18-(-22)$ & $-18-(-22)$ & $+13-(+26)$ & $+8-(+25)$ \\
\hline Strong & $>(-95)$ & $>(-95)$ & $>(-22)$ & $>(-22)$ & $>(+26)$ & $>(+25)$ \\
\hline $\begin{array}{l}\text { Distance from } \\
\text { the surface }\end{array}$ & $0-20$ & $20-40$ & $0-20$ & $20-40$ & $0-20$ & $20-40$ \\
\hline
\end{tabular}

making rocks). The main indicator in the diagnostic tables is the infiltration coefficient; porosity and bulk density are optional. The benchmarks are the humus content in soil determined by the Zaslavsky method (1979), as well as morphological parameters determined according to the method developed by Sobolev (1954).

\section{CONCLUSIONS}

This study has shown the principles of the possibility of application of the infiltration coefficient and porosity, as a specific field method for the study of aerodynamic similarity degree of erosion of soil. The advantage over traditional methods is the more precise gradation levels of erosion, based on a precise quantitative indicators rather than visual morphological characteristics. With certain adaptation and validation in the field, this method is applicable to diagnose the degree of erosion of zonal soils of other territories.

\section{REFERENCES}

Dullien, F.A.L. (1991) Porous Media, Second Edition: Fluid Transport and Pore Structure. Academic Press.

Ghanbarian, B., et al. (2013) Percolation theory generates a physically based description of tortuosity in saturated and unsaturated porous media. Soil Science Society of America Journal 77(6), 1920-1929, doi:10.2136/sssaj2013.01.0089.

Kraus, G., Ross, J. \& Gerifalco, L.A. (1953) J. Phys. Chem. 57, 212-226.

Onishchenko, V.G. (1986) Similarity of the thermodynamic characteristics of soil moisture and their general description. L., Autoabstract of the dissertation. 46.

Richards, L.A. (1928) The usefulness of capillary potential to soil moisture and plant investigations. J. Agr. Res. 37.

Sirotkin, V.M., Ginzburg, M.R. (1971) On determination of the specific surface of dispersion medium. Eurasian Soil Science 2. $108-116$.

Sirotkin, V.M., Sirotkin, V.V. \& Vasyukov S.V. (2012) Evaluation of Soil Hydrophysical Methods. Kazan (Volga Region) Federal University. Kazan: ID "MeDDoK", 220 p.

Sobolev, S.M. (1954) Nomenclature eroded (eroded) soils // Soil investigation and preparation of soil maps. $43-64$.

Stewart, R.D., et al. (2013) Non-destructive quantification of macropore volume using shear-thinning fluid. Soil Science Society of America Journal. 10.2136/sssaj2013.08.0346.

Zaslavsky, M.N. (1979) Soil Erosion, 245 p. 